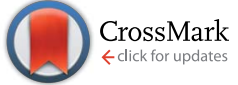

Cite this: RSC Adv., 2017, 7, 1749
Received 9th November 2016 Accepted 3rd December 2016

DOI: 10.1039/c6ra26561h

www.rsc.org/advances

\section{Dual delivery of chloramphenicol and essential oil by poly- $\varepsilon$-caprolactone-Pluronic nanocapsules to treat MRSA-Candida co-infected chronic burn wounds $\dagger$}

\begin{abstract}
Sanjeeb Kalita, ${ }^{\star a}$ Raghuram Kandimalla, ${ }^{a}$ Banasmita Devi, ${ }^{a}$ Bhupalee Kalita, ${ }^{\text {b }}$ Kasturi Kalita, ${ }^{c}$ Manab Deka, ${ }^{d}$ Amal Chandra Kataki, ${ }^{e}$ Arup Sharma ${ }^{f}$ and Jibon Kotoky ${ }^{\star a}$

Methicillin-resistant Staphylococcus aureus (MRSA) and Candida co-infections in the context of burn wounds, leads to morbidity and mortality. Eradication of such difficult to treat complex infection needs concomitant effective topical administration of an antibiotic-antifungal cocktail. Application of the broad-spectrum antibiotic chloramphenicol (CAM) has been hindered owing to its hydrophobicity, poor dermal penetration, rapid degradation and toxicity issues. But, due to its lesser uses in the past, CAM continues to be active against a majority of currently predominant resistant bacterial strains. Additionally, the essential oil of Cymbopogon flexuous possesses strong, broad spectrum antimicrobial property and also acts as a synergistic enhancer when used in combination with antibiotics. But due to its highly volatile nature and instability in free form, topical administration has not been possible. Keeping this in mind, we co-encapsulate CAM with lemongrass essential oil in PCL-Pluronic composite nanocapsules (CAM-LEO-PCL-P NCS) and were successful in overcoming the limitations in drug administration and achieved a significant reduction in cytotoxicity than that of the free counterparts with increased therapeutic index. CAM-LEO-PCL-P NCs exhibited significantly enhanced in vitro antimicrobial activity against 22 microbial pathogens including ten clinical MRSA isolates and three Candida species. This therapeutic nanocapsule was able to penetrate into burn wounds and demonstrated pronounced wound healing ability against MRSA-Candida co-infected burn wounds of mice by significant reduction of the pathogen burden. It also helps in increased cellular proliferation and collagen synthesis in the wounded area with the reduction of inflammatory cytokines. We propose CAM-LEO-PCL-P NCS as a potential candidate to treat chronic wound infections.
\end{abstract}

\section{Introduction}

Regardless of the developments in the management of burn injury, bacterial and fungal infections in burn wounds are still regarded as one of the most common and traumatic conditions which lead to significant mortality and morbidity. ${ }^{1}$ Burn

${ }^{a}$ Drug Discovery Lab, Institute of Advanced Study in Science and Technology, Guwahati - 781035, Assam, India. E-mail: sanju.kalita@gmail.com; jkotoky@gmail.com; Tel: +91-9957585400; +91-9435197316

${ }^{b} J a w a h a r l a l$ Nehru Centre for Advanced Scientific Research, Jakkur, Bengaluru, Karnataka 560064, India

'Saharia's Path Lab \& Blood Bank, Bhangagarh, Guwahati - 781005, Assam, India ${ }^{d}$ Department of Bioengineering and Technology, Gauhati University, Guwahati 781014, Assam, India

${ }^{e}$ Dr. B. Borooah Cancer Institute, Guwahati - 781016, Assam, India

${ }^{f}$ College of Veterinary Science, Assam Agriculture University, Guwahati - 781022, Assam, India

$\dagger$ Electronic supplementary information (ESI) available. See DOI: $10.1039 / \mathrm{c} 6 \mathrm{ra} 26561 \mathrm{~h}$ patients are susceptible to severe infectious complications like bacteraemia, sepsis or multiple organ dysfunction syndromes due to contributory critical factors like thermal destruction mediated avascularity of the eschar, destruction of the skin barrier, suppression of regional and systemic host humoral and cellular immune responses. The presence of coagulated proteins and other microbial nutrients in the wound also helps in this regard. ${ }^{2}$ Staphylococcus aureus, Pseudomonas aeruginosa, methicillin-resistant Staphylococcus aureus (MRSA) and Candida albicans are the prevalent microbial infections that occur in patients immunocompromised with their burns. ${ }^{3}$ MRSA constitutes $40 \%$ of wound isolates and infects the wounds of $14-17 \%$ of thermally injured patients once they were colonized. ${ }^{4}$ In a study of 300 patients, it was found that; burn wounds were co-infected by Candida species in 59\% of cases. Candida species co-infected burn wounds with bacteria. ${ }^{5}$ These mix population of microorganisms forms multi-species biofilms within 48-72 hours of injury at the burn site. These infections are difficult to treat with antibiotics and antifungals as in the mixed infections, 
the antibiotic resistance profiles changes over time. ${ }^{1}$ C. albicans assist the invasion of $S$. aureus across mucosal barriers, which ultimately lead to systemic infection. ${ }^{6}$ Even $C$. albicans can provoke a systemic infection through the morphological transformation from the round shaped yeast to the invasive hyphal form. MRSA infections make the body more vulnerable to other types of bacterial and fungal infections and ultimately lead to the establishment of multi-species wound infections which are very difficult to eradicate. ${ }^{7}$ S. aureus and MRSA can also remain hidden inside the epithelial cells as L-forms and bio films long after the initial infection has cleared, and in favourable conditions, the bacteria will again initiate fresh and more complex infections. To treat this serious MRSA and fungal co-infections we need an integrated therapeutic agent which can not only effectively kill MRSA infections but also can eradicate subsequent candidiasis of the burn wound. But there is not even a single broad spectrum drug candidate available to cure this difficult to treat infections. The scarcity of effective treatment options forced health care professionals to reintroduce unremembered antibiotics into their clinical practice.

For the treatment of chronic dermal and sub dermal wound infections; US Food and Drug Administration approved therapeutics are not available till date. ${ }^{3}$ Few old antibiotics have remained functional against a significant portion of presently prevalent bacterial pathogens due to their low-level use in the past. An old antibiotic chloramphenicol (CAM) exhibited bactericidal activity against clinical isolates of MRSA with minimum inhibitory concentrations (MICs) of $8 \mu \mathrm{g} \mathrm{ml} \mathrm{m}^{-1} \cdot{ }^{8}$ Though previously it was considered that CAM is less effective as well as more toxic compared to newer agents, now clinicians are reassessing their use in various patient populations with difficult to treat infections. On the other hand, essential oils are reported to have strong broad-spectrum antimicrobial activity as well as acts as synergistic enhancers. The combinatory effect shown by the essential oil in combination with antibiotics transcend their individual performance resulted in the reduction in the minimum effective dose with reduced adverse side effects of the antibiotic. Essential oils are reported to exhibit its activity mainly through $\beta$-lactamase inhibition, bacterial efflux pump inhibition, cell wall and membrane disturbance and antiquorum sensing. ${ }^{9}$ To cope up with bacterial resistance, essential oil exploitation is believed to be more promising as it is multicomponent in nature compared to other common antimicrobials that only possess a particular target site. ${ }^{\mathbf{1 0}}$

In this regard, to address the toxicity and other barriers like stability, bioavailability in clinical uses of this therapeutic agent, nanoparticulate delivery can be the solution. Polymeric nanoparticulate biodegradable drug delivery system helps to achieve better penetration, sustained release, better bioavailability, stability and the pharmacokinetic parameters of therapeutic entities with a significant increment in the therapeutic index; ultimately leads to lowered levels of toxicity compared to the crude drug. ${ }^{11}$

Although anti-MRSA properties of several nanoscopic systems has been evaluated, only our group has reported polymeric nanoparticulate system encapsulated CAM for the effective treatment of MRSA infected burn wound conditions.
Though our reported system demonstrated significant efficacy, we were still in search of a better option having not only antibacterial/anti-MRSA activity but also should possess broad spectrum antifungal activity along with superior wound healing property. In this purpose, we have chosen CAM (CAM), as a broad-spectrum bacteriostatic antibiotic which stops bacterial growth by blocking the peptidyl transferase activity of the bacterial ribosome and thereby inhibiting protein chain elongation of bacterial cell wall synthesis. Due to its broad spectrum effectiveness with excellent blood-brain barrier penetration capability, it has been used as the first choice for treatment of vancomycin-resistant Enterococcus infections and Staphylococcal brain abscesses. But the intravenous and oral administration of CAM leads to serious side effects like bone marrow toxicity, hypersensitivity reactions, etc. Even so, due to the frequent occurrence of antibiotic resistance; CAM has still been utilized by the clinicians in recent years.

One of the major hurdles in the treatment of thermal wound infection is the unavailability of systemic therapeutics due to avascularity of the burn eschar. In this regard, topical antimicrobials are preferable for effective therapy of site-specific superficial or invasive infections due to its intrinsic ability to evade systemic toxicity and the ease of site-specific rapid delivery. ${ }^{12}$ For the treatment of MRSA-infected burn wounds, only a few treatment options are available (Eg: Moxifloxacin, wound dressing of nanocrystalline silver); which therapeutic efficacy is hindered by insufficient eschar penetration ability and toxicity issues. ${ }^{1}$ Furthermore, the hydrophobic chemical structure of CAM obstructs adequate cutaneous penetration and hence it is not used for topical treatment of dermal infections.

Nanocapsules along with other nanometric systems possess a great surface area, which renders them highly satisfactory for the topical application of lipophilic substances assisting a sustained drug release. ${ }^{13}$

We have used biocompatible, biodegradable, non-immunogenic polymer; poly( $\varepsilon$-caprolactone) (PCL) as an encapsulating agent. PCL is the highly explored polymer for subcutaneous drug delivery systems due to its high physical stability and simple preparation methods. To facilitate the solubilization of lipophilic therapeutic molecules in physiological media, a biocompatible nonionic surfactant polyol (molecular weight approximately $12500 \mathrm{Da}$ ), Pluronic F127 (PF127) is used. ${ }^{14}$

In this present research effort, we for the first time suggest a drug delivery model system that co-encapsulate antibiotic with essential oil in a polymeric nanoparticulate system for achieving the synergistic effect with higher therapeutic index. CAM dissolved into lemon grass essential oil and coencapsulated into PCL-Pluronic composite nanocapsule (NCs) should intensify subcutaneous penetration and sustained release of both CAM and lemongrass essential oil. The utility of essential oil as skin penetration enhancer is already reported. ${ }^{15}$ This system will also protect the essential oil from volatilization and maintain its functional stability. We also hypothesize that encapsulation results in more direct and prolonged contact between the antibiotic and MRSA, antibiotic resistance reversal by essential oil and thereby increased therapeutic index with 
reduced level of toxicity. Along with significant broad spectrum antimicrobial activity, essential oils also possess antiinflammatory efficacy which will help in reduction of inflammation resulted from burn and infections. ${ }^{16,17}$ Furthermore, we believe the successful application of this nanoparticulate system will effectively eradicate MRSA and Candida albicans infections of the burn wounds resulted in superior healing efficacy.

\section{Materials and methods}

A detailed description of the materials and methodology used in this study is provided in the ESI. $\dagger$

\subsection{Ethics statement}

Animal experimentation protocols were approved by the Institutional Animal Ethical Committee (CPCSEA registration number: $1706 / \mathrm{GO} / \mathrm{c} / 13 / \mathrm{CPCSEA})$ at the Institute of Advanced Study in Science and Technology, Guwahati-35 (IASST/IAEC/ 2015-16/801). CPCSEA: Committee for the purpose of control and supervision of experiments on animals. For experiments on human blood cells, an informed consent was obtained from the human volunteer of this study.

\section{Results}

\subsection{Chemical components of LEO}

Hydro-distillation of Cymbopogon flexuous leaves yielded 4\% essential oil. A total of 82 compounds with 12 major compounds are identified with principle compounds viz. geraniol, neral, $\alpha$ phellandrene and linalool (Table S1 $\dagger$ ). The chemical composition of LEO was mainly composed of terpenoids, monoterpenes, and monoterpenols.

\subsection{FE-SEM, particle size and zeta potential analysis of CAM-LEO-PCL-P NCS}

The lemongrass essential oil used as co-solvent assisted in solubility of CAM and thus got internalized into the hydrophobic core of PCL-P NCs. As CAM has different solubility in different organic solvents, encapsulation of CAM into the PCLNCs was greatly affected by the presence or absence of a cosolvent. Spherical solid, dense, structured particles with average size of $105 \mathrm{~nm}$ were formed as observed under FE-SEM (Fig. 1A and B). Dynamic light scattering measurements exhibited hydrodynamic size of CAM-LEO-PCL-P NCs was 124.8 whereas LEO-PCL-P NCs showed particle size of $123.5 \mathrm{~nm}$. The LEO-PCL-Pluronic nanocapsules demonstrated a negative surface charge of $-29.6 \mathrm{mV}$ whereas the CAM-LEOPCL-P NCs showed zeta potential value of $-22.4 \mathrm{mV}$.

\subsection{X-ray diffraction analysis}

CAM extracted from CAM-LEO-PCL-P NCs were found to maintain its crystalline nature as confirmed by the similarity in diffraction patterns of isolated CAM to pure CAM ( $2 \theta$ at 7.96, 8.43, 7.96, 8.90, 14.54, 17.24, 11.76, 14.14, 16.91, 17.87, 20.80, 23.10, 24.21, 25.24, 26.98, 27.86 and 28.65) (Fig. 1C). PCL also maintained its semi-crystalline nature (peaks at about 23.7 and
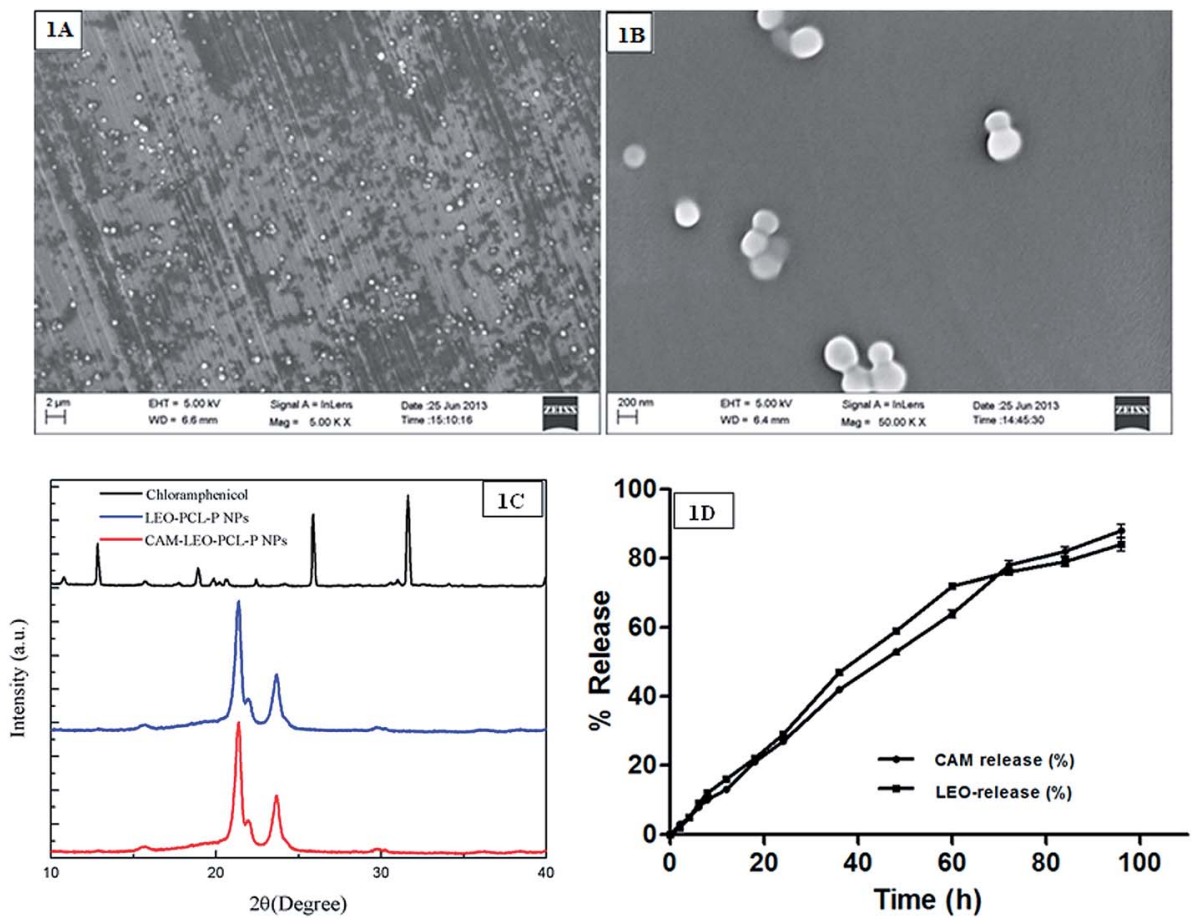

Fig. 1 Images of CAM-LEO-PCL-P NCs viewed under field emission scanning electron microscopy. Notes: (A) CAM-LEO-PCL-P NCs at 5.00kX magnification; (B) CAM-LEO-PCL-P NCs at $50.00 \mathrm{k} \times$ magnification. (C) X-ray diffraction spectra of CAM, LEO-PCL-P NCs, and CAM-LEO-PCL-P NCs. (D) In vitro CAM release (\%) and LEO release (\%) from CAM-LEO-PCL-P NCs. Note: released in phosphate-buffered saline (pH 7.4) at $37{ }^{\circ} \mathrm{C}$. 
21.4) during the fabrication process, as the LEO-PCL-P NCs and CAM-LEO-PCL-P NCs exhibited an identical type of Bragg peaks with that of the pure PCL. As characteristics brag peaks of CAM was not observed in the CAM-LEO-PCL-P NCs, which suggest that significant portion of the CAM is got incorporated inside the NCs but not interacted onto the NCs' surface of the NCs.

\subsection{Encapsulation efficiency (EE\%)}

The EE of CAM in PCL-P NCs was controlled by the drug to polymer ratio (Table $\mathrm{S} 2 \dagger$ ). In ten different reaction mixtures, $62.5 \mathrm{mg}$ of PCL concentration was kept as constant, but the concentration of drug differed. Highest EE (\%) of $98.3 \%$ was obtained when the drug to PCL ratio (w/w) was $7.5: 62.5$ i.e. $7.3725 \mu \mathrm{g}$ of CAM got encapsulated. The EE increased as we increased drug concentration until it attained a plateau at $7.5 \mathrm{mg}$ of CAM and then started decreasing. EE\% changes along with the added CAM amount confirmed the successful encapsulation of CAM into PCL-P NCs. It is observed that EE of the prepared NCs got significantly increased upon the use of PF127 (data not shown). These phenomena can be attributed to selfassembly property of the PF127 in the aqueous environment where CAM got encapsulated by the hydrophobic core and hydrophilic parts interacted with PCL.

The concentration of the lemongrass essential oil was estimated using a standard graph, $y=0.0823 x+2.3884, R^{2}=$ 0.9756. Samples were analyzed in triplicates, and it was found that $86.9 \%$ of the lemongrass oil was encapsulated into the NCs.

\subsection{In vitro drug release}

In vitro CAM release profile in the first 96 hours is evaluated (Fig. 1D). 13\% of CAM was released in first 12 hours, which slowly increased up to $27 \%$ in 24 hours. Subsequently, a drop in the rate of release was noticed, and finally $88 \%$ of CAM was released in up to $96 \mathrm{~h}$. It is observed that CAM got released from the nanocapsule system in a sustained manner whereas the $t_{50}$ (time taken for $50 \%$ drug release) was calculated as $48 \mathrm{~h}$ (53\%).

\subsection{In vitro lemongrass essential oil release}

LEO exhibited a controlled release profile up to $60 \mathrm{~h}$ with $72 \%$ release; further, it slows down, and a total of $84 \%$ of LEO got released in $96 \mathrm{~h}$ (Fig. 1D). The nanocapsule system gives sustained prolonged release of LEO which is advantageous for topical drug delivery. The CAM dissolved in LEO also exhibited a prolonged and sustained release which is a prerequisite for therapy of chronic dermal wounds.

\subsection{Release kinetics}

The overlay plot of LEO and CAM release (\%) is depicted in Fig. 1D. It reveals that increase in CAM release was influenced by the consequential increase in LEO release $(r=0.993, p<$ 0.001). The correlation can be obtained by following the best fitted polynomial fit degree $=3$ model.

CAM release $(\%)=1.504+0.829 \times$ LEO release profile $(\%)+$ $0.003 \times($ LEO release profile $(\%)-36.571) 2+0.0001087 \times($ LEO release profile $(\%)-36.5714) 3\left(R^{2}=99.61 \%\right)$. It further reveals it is best fitted with release kinetic data of CAM and LEO. Individually, release kinetics data of both CAM and LEO was best fitted with polynomial fit degree $=4\left(R^{2}=0.998244\right.$ for LEO and $R^{2}=0.998958$ for CAM).

\subsection{Biocompatibility studies}

3.8.1. Hemolytic activity. Low to mild hemolytic activity was observed in the case of CAM-LEO-PCL-P NCs, free CAM and free LEO treated human erythrocytes. The hemolytic assay was performed in triplicate and results are expressed in \% hemolysis as a mean \pm standard deviation. The result suggested that the CAM-LEO-PCL-P NCs (at dose $1000 \mu \mathrm{g} \mathrm{ml}^{-1}$ ) causes lesser hemolysis $(1.89 \% \pm 0.12 \%)$ compared to free CAM $(6.63 \%$ $\pm 0.19 \%)$ and free LEO (4.18 $\pm 0.21 \%)$. An increase in percentage hemolysis was observed in a dose-dependent manner. CAM encapsulation in the polymeric nanocapsule attributed to an average of $36.19 \%$ lowering of hemolytic activity for all the tested concentrations of CAM-LEO-PCL-P NCs compared to free CAM.

3.8.2. Effects on RBC morphology. The mature RBCs shows the shape of biconcave discs which alter upon interaction with membrane active toxic substances. FESEM images revealed that the encapsulation of CAM and LEO in PCL-P nano capsular system significantly reduced the alterations or deformities as compared to free CAM and free LEO (Fig. 2). This happens due to the protection effect of PCL-P NCs which obstruct direct interaction between CAM/LEO and RBCs. Whereas the distilled water treated RBCs showed complete lysis and destruction and PBS-treated samples maintained normal morphology.

3.8.3. Effects on $\mathrm{RBC}$ and $\mathrm{WBC}$ count. The results of $\mathrm{RBC}$ and WBC count post $3 \mathrm{~h}$ incubation with free CAM, free LEO and CAM-LEO-PCL-P NCs is depicted in Table S3. $\dagger$ Blood treated with free CAM and free LEO showed decrease in both RBC and WBC count whereas CAM-LEO-PCL-P NCs treatment showed far better results. But, no significant difference was observed between treated groups and negative control.

3.8.4. Cytotoxicity study results of free CAM, free-LEO, LEO-PCL-P NCs, and CAM-LEO-PCL-P NCs. $24 \mathrm{~h}$ of post treatment with CAM-LEO-PCL-P NCs $\left(250 \mu \mathrm{g} \mathrm{ml}^{-1}\right)$ on L929 mouse skin fibroblastic cells and human dermal fibroblastic (HDF) cells demonstrated a much higher viabilities $(83 \%$ and $86 \%)$ than free CAM $(73 \%$ and $78 \%$ ) and free LEO (76\% and $80 \%$ ) respectively (equilibrated concentration). Colony forming assay results reveal that CAM-LEO-PCL-P NCs (up to $250 \mu \mathrm{g}$ $\mathrm{ml}^{-1}$ for $48 \mathrm{~h}$ ) does not resulted in significant cytotoxicity against L929 and HDF cell lines which evident from survival fraction values of 91.4 and 93.3 respectively. Whereas free CAM and free LEO treated sample showed survival fraction of 81.3, 84.2 and 82.8, 86.5 respectively towards L929 and HDF cell lines.

\subsection{In vitro antimicrobial activity}

In case of nanocapsule applications, retention of therapeutic efficacy of the encapsulated drug is a major concern since denaturation and structural modification may occur in the fabrication process as well as in the course of release. Zone of inhibition, MIC, MBC and time kill assay studies were 

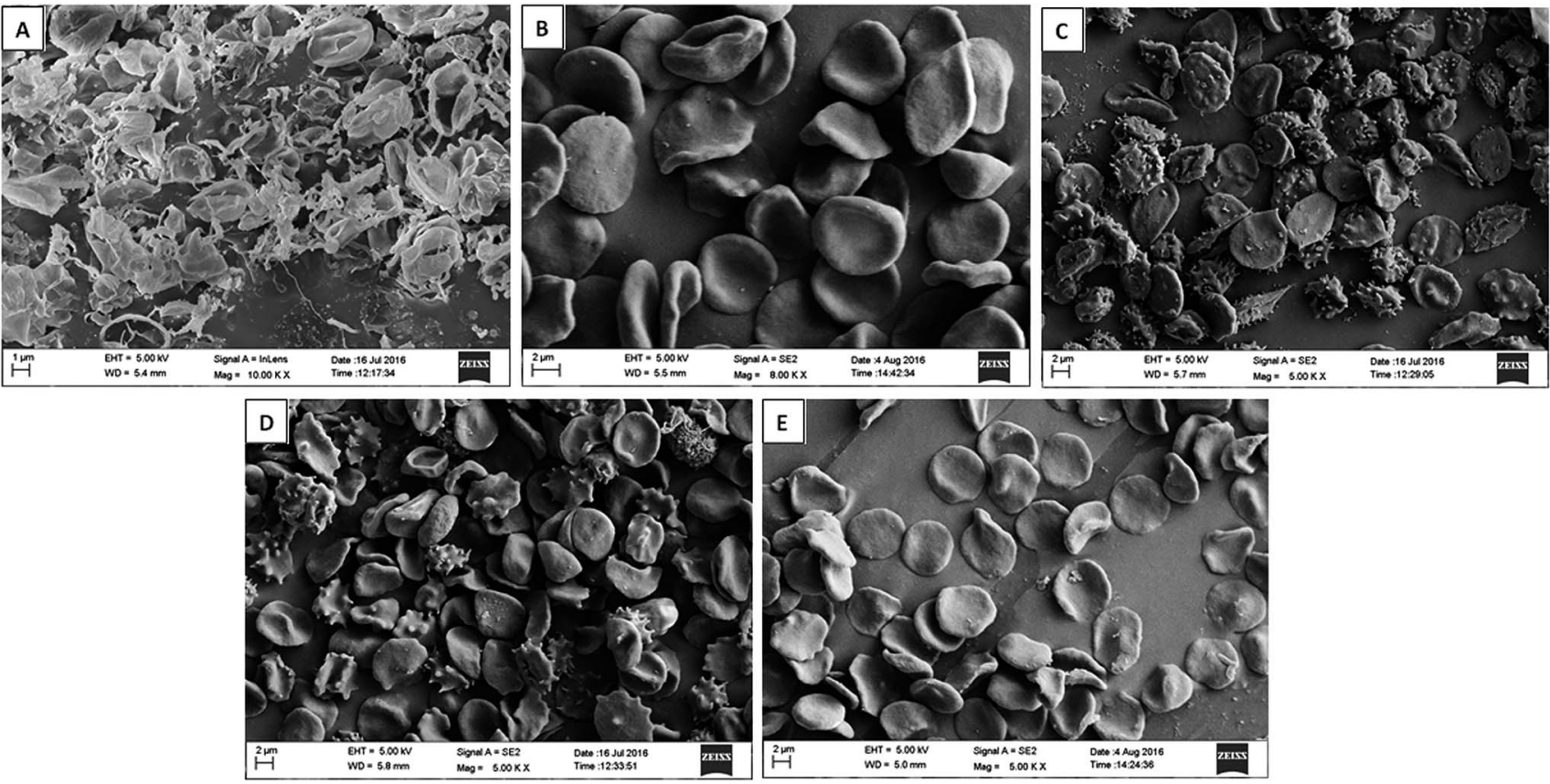

Fig. 2 Morphology of the RBCs in the presence of different treatments (A) distilled water, (B) phosphate buffer saline, (C) free CAM, (D) free LEO, (E) CAM-LEO-PCL-P NCs under scanning electron microscope.

performed to investigate the enhanced antibacterial action of the novel CAM-LEO-PCL-P NCs in comparison to free CAM and free LEO. The result of zone of inhibition experiment (Table $\mathrm{S} 4{ }^{\dagger}$ ) clearly demonstrates the encapsulation of CAM in LEOPCL-P NCs. The nano encapsulation system augments CAM antibacterial activity and showed greater zone of inhibition against the nine tested bacterial strains. The most significant observation is that, CAM-LEO-PCL-P-NCs exhibit synergistic activity against ten number of MRSA clinical isolates whereas free CAM and free LEO exhibited a lesser diameter of zone of inhibition (Table S5 $\dagger$ ). From all the zone of inhibition data it is evident that due to increased solubility of CAM, enhanced stability of LEO and synergism effect of both the agents CAMLEO-PCL-P NCs exhibited superior activity against the tested pathogenic bacteria including MRSA. The MIC/MBC values of the CAM-LEO-PCL-P NCs against the tested bacteria (Table S6 $\dagger$ ) including MRSA strains (Table 1) was ranged from 2 to 12 $\mu \mathrm{g} \mathrm{ml}{ }^{-1}$, which is significantly lower than the MIC/MBC values of free CAM and free LEO. MIC $\leq 32 \mu \mathrm{g} \mathrm{ml}{ }^{-1}$ as resistant $\geq 16 \mu \mathrm{g}$ $\mathrm{ml}^{-1}$ as intermediate and $\geq 8 \mu \mathrm{g} \mathrm{ml}^{-1}$ was taken as susceptible as per the Clinical and Laboratory Standards Institute recommendations. Though, LEO is regarded as a potent anti Candida agent, due to its volatilization and other stability issues, it is not that effective in topical administration against superficial chronic wound conditions. Encapsulation of LEO in PCL-P NCs significantly increases the anti Candida activity as evident from results of zone of inhibition (Table S7 $\dagger$ ). The nano encapsulation system showed highest activity against $C$. albicans and lowest against $C$. glabrata. The MIC/MFC values of the CAMLEO-PCL-P NCs against the tested Candida species ranged from 6 to $24 \mu \mathrm{g} \mathrm{ml}^{-1}$, whereas the free LEO showed MIC/MFC values raging from 48 to $96 \mu \mathrm{g} \mathrm{ml}{ }^{-1}$ (Table S8 $\dagger$ ). The CAM-
LEO-PCL-P NCs was able to impede the biofilm formation by $95.2 \%, 96.7 \%, 94.3 \%$ and $88.2 \%$ respectively for the tested $S$. aureus, $P$. aeruginosa, MRSA 1, MRSA 2 strains whereas free CAM can able to exhibit inhibition of the same strains by $72.8 \%$, $83.2 \%, 79.1 \%$ and $70.7 \%$ respectively. Free LEO exhibited much poor inhibition against the biofilm formation and exhibited only $44.2 \%, 56.7 \%, 41.9 \%$ and $39 \%$. Since MRSA 1 and MRSA 2 showed highest susceptibility towards CAM-LEO-PCL-P NCs, these two strains were taken for further evaluate the killing kinetics. CAM-LEO-PCL-P NCs showed increased killing of MRSA 1 and MRSA 2 compared to free CAM and free LEO in 4, 8 and $12 \mathrm{~h}$ time points (Fig. 3B). Whereas the non-treated MRSA

Table 1 MIC/MBC values of CAM-LEO-PCL-P NCs, free CAM and free $L E O$ tested against ten number of clinical MRSA isolates ${ }^{a}$

\begin{tabular}{lllll}
\hline S. no & $\begin{array}{l}\text { Test bacterial } \\
\text { strains }\end{array}$ & $\begin{array}{l}\text { Free CAM } \\
\left(\mu \mathrm{g} \mathrm{ml}^{-1}\right)\end{array}$ & $\begin{array}{l}\text { Free LEO } \\
\left(\mu \mathrm{g} \mathrm{ml} l^{-1}\right)\end{array}$ & $\begin{array}{l}\text { CAM-LEO-PCL-P } \\
\mathrm{NCs}\left(\mu \mathrm{g} \mathrm{ml} l^{-1}\right)\end{array}$ \\
\hline 1 & MRSA 1 & $32 / 32$ & $128 / 256$ & $2 / 4$ \\
2 & MRSA 2 & $12 / 16$ & $128 / 128$ & $2 / 4$ \\
3 & MRSA 3 & $12 / 16$ & $64 / 258$ & $2 / 4$ \\
4 & MRSA 4 & $8 / 8$ & $64 / 128$ & $2 / 2$ \\
5 & MRSA 5 & $8 / 8$ & $64 / 128$ & $2 / 4$ \\
6 & MRSA 6 & $8 / 8$ & $128 / 256$ & $4 / 4$ \\
7 & MRSA 7 & $20 / 24$ & $256 / 256$ & $4 / 8$ \\
8 & MRSA 8 & $16 / 20$ & $128 / 128$ & $4 / 8$ \\
9 & MRSA 9 & $12 / 16$ & $128 / 128$ & $4 / 4$ \\
10 & MRSA 10 & $8 / 12$ & $64 / 128$ & $4 / 8$
\end{tabular}

a Abbreviations: CAM, chloramphenicol; CAM-LEO-PCL-P NCs, chloramphenicol dissolved in lemongrass essential oil loaded with poly(e-caprolactone)-Pluronic composite nanocapsules; MIC, minimum inhibitory concentrations; MBC, minimum bactericidal concentrations; MRSA, methicillin-resistant Staphylococcus aureus. 
demonstrates significant increase of the CFU count in all the tested time points. The difference between the CAM-LEO-PCL$P$ NCs, free-CAM and free LEO groups remained significant for the dose of $32 \mu \mathrm{g} \mathrm{ml}^{-1}$ against the tested MRSA strains. This superior killing rate is helpful in impeding biofilm formation efficiently. As well as, CAM-LEO PCL-P NCs also demonstrated significant enhanced killing of $C$. albicans as compared to free LEO in 6, 12 and $24 \mathrm{~h}$ time points (Fig. 3A).

\subsection{In vivo experimental results}

3.10.1. Microbial burden or CFU count in in vivo experiment. The in vivo performance of the CAM-LEO-PCL-P NCs exhibit a significant decrease in the MRSA and C. albicans CFU burden compared to free CAM and free LEO treated groups at days 3, 7, 11 and 15 (Fig. 4A and B). CAM-LEO-PCL-P NCs demonstrated significantly better results at MRSA and
C. albicans killing as well as demonstrated enhanced wound closure efficacy in compared to the concentration-equilibrated free CAM and free LEO-solution group at different time intervals (Fig. 5).

3.10.2. Immunological response to burn injury. Infections on burn wound leads to elevated levels of tumor necrosis factor alpha and proinflammatory cytokines Interleukin-1 $\beta$. Though free CAM and free LEO reduces the level up to some extent but CAM-LEO-PCL-P NCs administration brought down the proinflammatory cytokines level comparable to normal animal. Concurrently, with the rise of proinflammatory cytokine levels, IL-6 levels also got increased and induce fever and leads to T-cell activation by the generation of acute-phase reactants. ${ }^{18}$ Accelerated rates of morbidity and mortality was associated with high levels of IL-6, hence it is also regarded as a marker for disease severity. On treatment with CAM-LEO-PCL-P NCs, significant reduction in IL-6 levels was observed compared to free CAM and
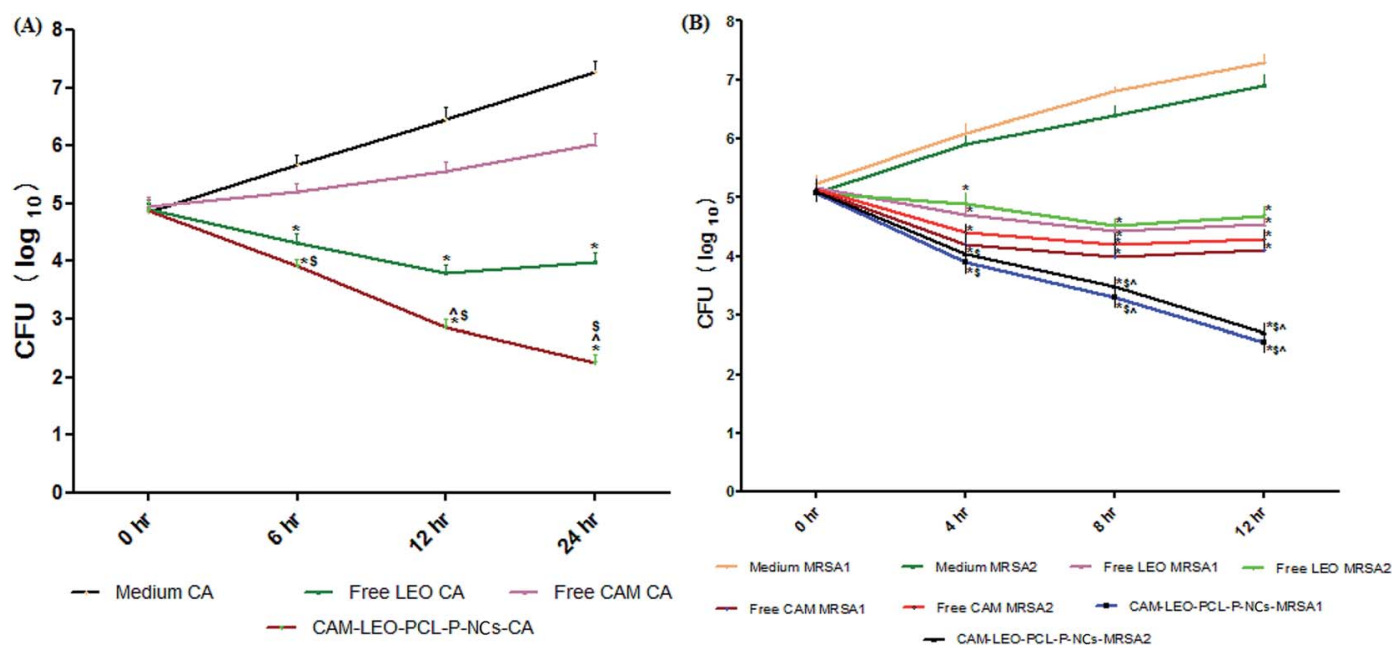

Fig. 3 (A) Time-kill assay of C. albicans incubated with media, free CAM, free LEO and CAM-LEO-PCL-P NCs at different time intervals. Notes: surviving CFUs at selected time points are shown. $* P \leq 0.05$, comparison of medium CA with all the other groups. $\$ \leq 0.05$, comparison of free CAM CA with CAM-LEO-PCL-P NCs-CA. ^ $\leq 0.05$, comparison of free LEO CA with CAM-LEO-PCL-P NCs-CA. (B) Time-kill assay of MRSA 1 and MRSA 2 incubated with media, free CAM, free LEO and CAM-LEO-PCL-P NCs at different time intervals. $* P \leq 0.05$, comparison of medium MRSA 1 \& MRSA 2 with all the other groups. $\$ \leq 0.05$, comparison of free LEO MRSA $1 \&$ free LEO MRSA 2 with CAM-LEO-PCL-P NCs-MRSA $1 \&$ CAM-LEO-PCL-P NCs-MRSA 2. ^ $\leq 0.05$, comparison of free LEO CAM with CAM-LEO-PCL-P NCs. $\wedge \leq 0.05$, comparison of free CAM MRSA $1 \&$ free CAM MRSA 2 with CAM-LEO-PCL-P NCs-MRSA 1 \& CAM-LEO-PCL-P NCs-MRSA 2.
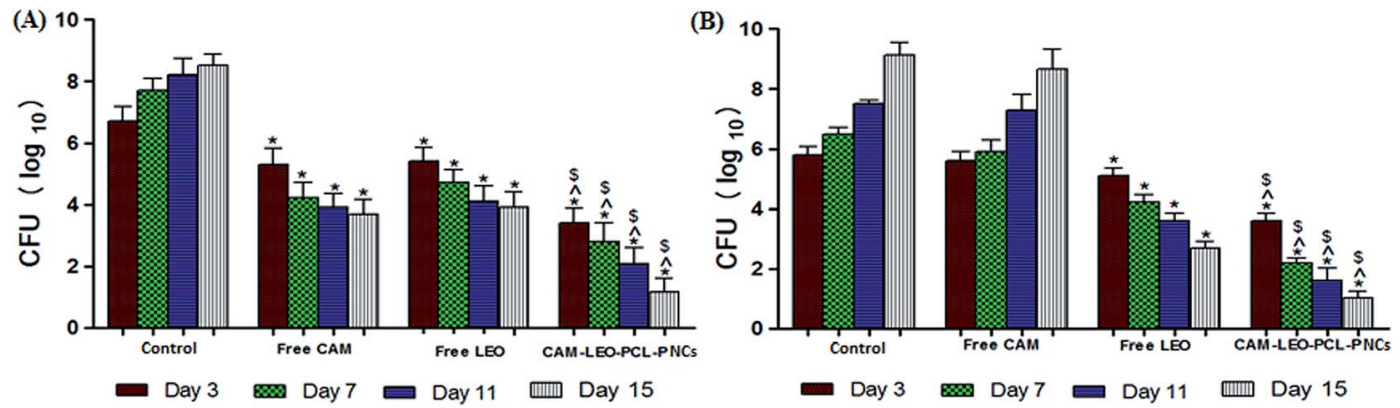

Fig. 4 (A) Effect of in vivo MRSA burden on topical application of free CAM, free LEO \& CAM-LEO PCL-P NCs at various time intervals ( $3^{\text {rd }}$, $7^{\text {th }}$, $11^{\text {th }}$ and $15^{\text {th }}$ day). (B) Effect of in vivo C. albicans burden on topical application of free CAM, free LEO \& CAM-LEO PCL-P NCS at various time intervals $\left(3^{\text {rd }}, 7^{\text {th }}, 11^{\text {th }}\right.$ and $15^{\text {th }}$ day). $* p \leq 0.05$ in comparison of control with all the other treatment groups. $\$ p \leq 0.05$ in comparison of free CAM with CAM-LEO-PCL-P NCs-CA. $\wedge \leq 0.05$, comparison of free LEO with CAM-LEO-PCL-P NCs-CA. 


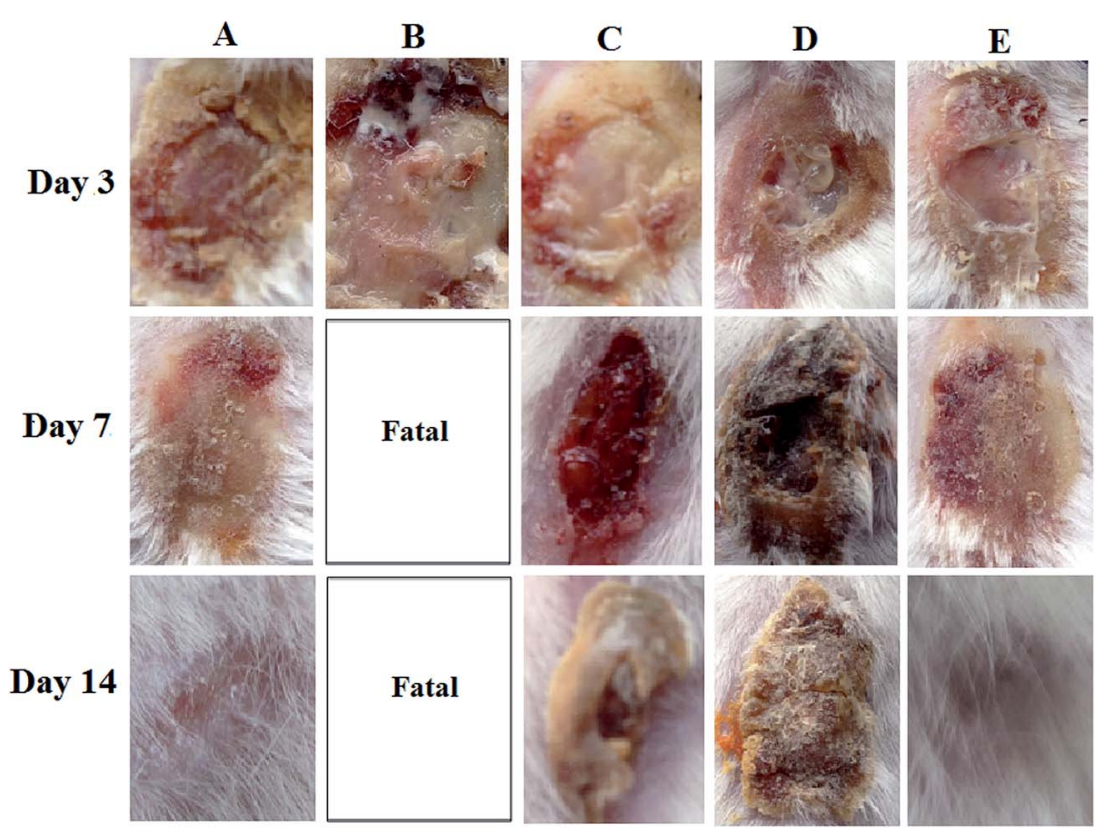

Fig. 5 MRSA-Candida albicans co-infected burn wound over time: (A) wounds with no infection and treatment; (B) wounds with infection and no treatment; (C) wounds with infection and treated with free CAM (D) wounds with infection and treated with free LEO (E) wounds with infection and treated with CAM-LEO-PCL-P NCs.

free LEO treatment. To circumvent the inflammation, $\mathrm{T}$ helper cells start differentiate into Th-2 cells, which generates the antiinflammatory cytokines IL-10. In this case, it was observed that, following CAM-LEO-PCL-P NCs administration, IL-10 levels got increased substantially as compared to free CAM, free LEO and nonrelated animals (Fig. 6A-D).

3.10.3. Hydroxyproline, hexosamine and uronic acid content. Estimation of hydroxyproline and hexosamine content
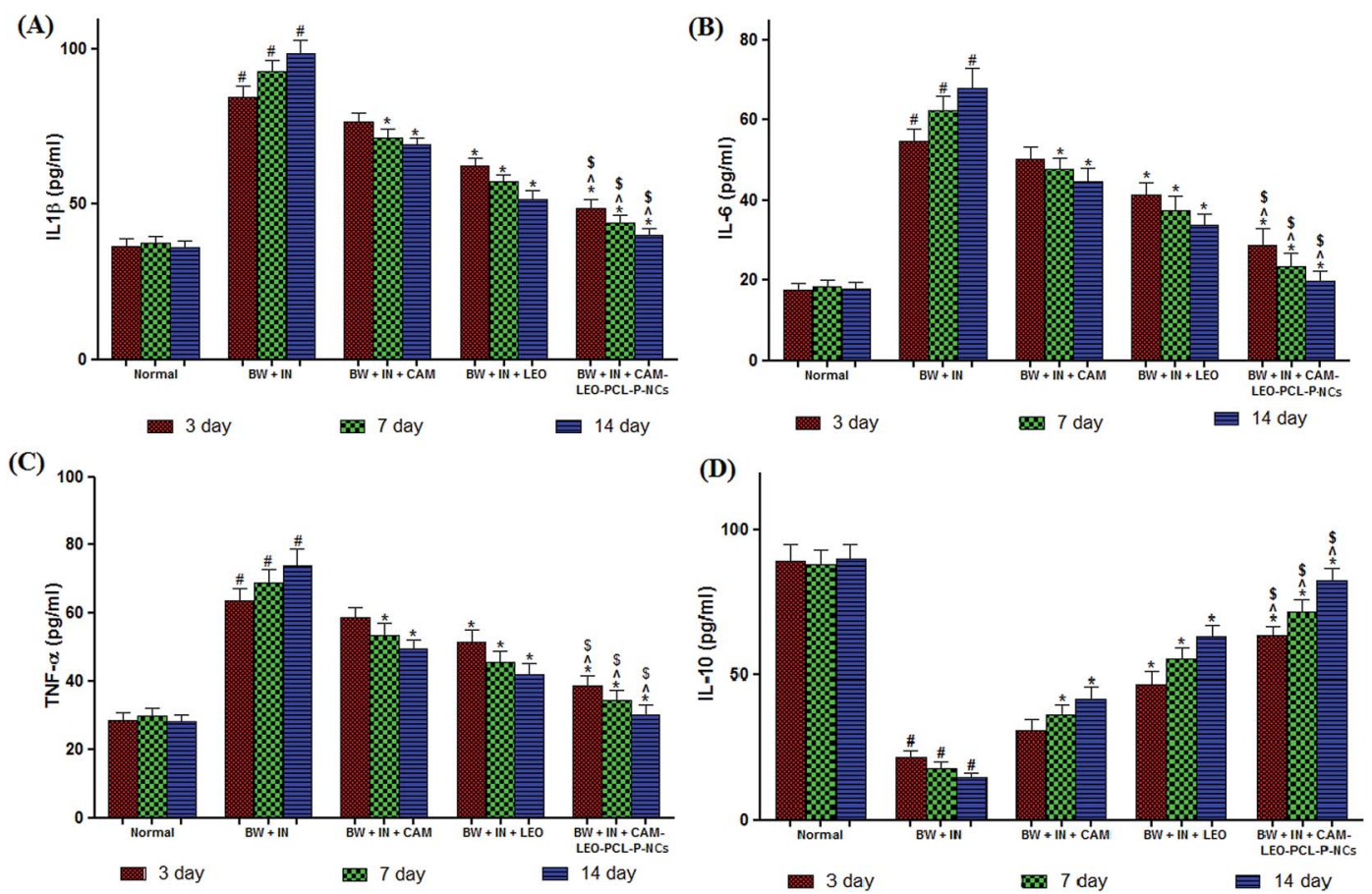

Fig. 6 Effect of various treatments on the tissue cytokine levels (A) IL-1 $\beta$; (B) IL-6; (C) TNF- $\alpha$; (D) IL-10 at different time intervals. All the results were expressed in mean $\pm S D$. $\# p \leq 0.05$ in comparison of animals with infection and no treatment with normal animals. * $p \leq 0.05$ in comparison of drug treated animals with non treated animals. $\$ p \leq 0.05$ in comparison of CAM-LEO-PCL-P-NPs treated animals with free CAM treated animals. ${ }^{\wedge} p \leq 0.05$ in comparison of CAM-LEO-PCL-P-NPs treated animals with free LEO treated animals. 
in the granulation tissues in control animal and free CAM, free LEO, CAM-LEO-PCL-P NCs treated animals reveals a significant increase in both hexosamine and hydroxyproline content in nanocapsule treated animals compared to the rest (Table S9†). There is gradual increase in hydroxyproline content on all the groups but control animals exhibit a very slow gradual increase till $15^{\text {th }}$ day of observation. It is observed that, hydroxyproline and hexosamine content were found to be significantly more in CAM-LEO-PCL-P NCs treated group though out the course of healing, which are important constituent of extracellular matrix for healing. Uronic acid content was also got significantly increased in the CAM-LEOPCL-P NCs treated animals than that of free CAM and free LEO treated and control animals (Table $\mathrm{S} 10 \dagger$ ). Increase in uronic acid level in treated animals compared to control animals suggests pronounced synthesis of glycosaminoglycans which is similar to earlier observations. ${ }^{19}$

3.10.4. Histopathology. Wound healing progression was evaluated on basis of histopathology analysis of tissue sections obtained on day $15^{\text {th }}$ to assess wound healing progression. In the case of the control burn on day three revealed, complete disruptions, serous crust, frank dermal necrosis, disorganized inflammation of epidermis, sub-epidermis and dermis layers with some bulbous elements were noted in the wound area. Where as in case of free LEO treated group, a little epidermal reepithelialization was observed with ulcerated epidermis, inflammatory granulation tissue and eschar was seen in the dermis. Furthermore, the free CAM treated group animals also exhibit a little amount of epidermal re-epithelialization with eschar and inflammatory granulation tissue was observed in the dermis. The CAM-LEO-PCL-P NCs treated group showed improved healing, where the ultrastructure was better organized with pronounced re-epithelialization with intact prominent

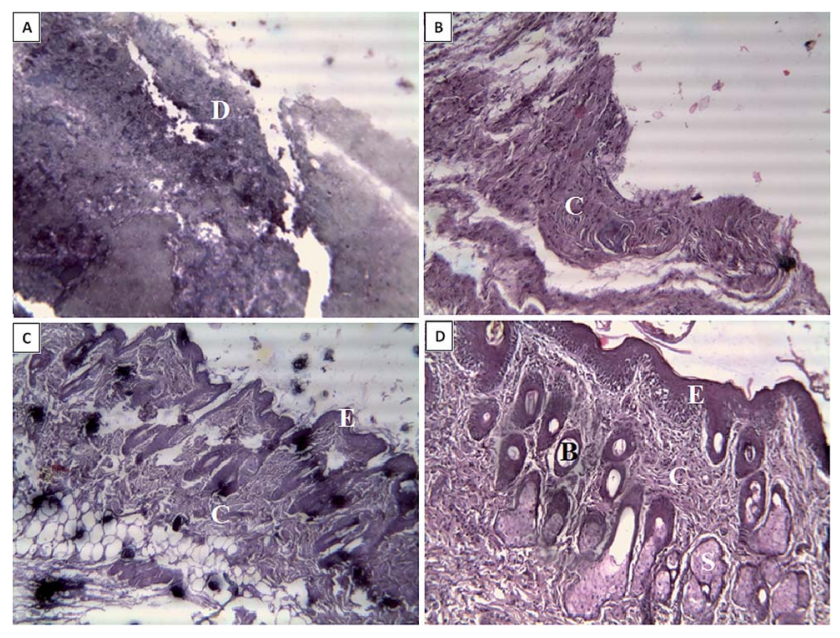

Fig. 7 Effect of different drug treatments on histopathology on wounds from MRSA-Candida albicans co infected burn wound from Swiss albino mice at $15^{\text {th }}$ day of the drug treatment. (A) Animals coinfected with MRSA-CA and no drug treatment (B) animals co-infected with MRSA-CA and treated with free-CAM (C) animals co-infected with MRSA-CA and treated with free-LEO (D) animals co-infected with MRSA-CA and treated with CAM-LEO-PCL-P NCS. acanthotic maturing sub-epidermis and dermis along with normal skin adnexal structures. Based on a quantitative and qualitative clinical analysis, CAM-LEO-PCL-P NCs treatment lead to decreased tissue induration, necrotic crusting, peripheral wound erythema and edema in comparison to infected control animal group (Fig. 7).

\subsection{Storage stability}

Nanocapsule suspensions maintained its initial characteristics with respect to encapsulation efficiency and size after six months of storage at 4 and $25{ }^{\circ} \mathrm{C}$, respectively $(p<0.05)$.

\section{Discussion}

In spite of the developments in the treatment strategies, MRSA and Candida sp. infected chronic burn wounds cause severe traumatic conditions, leads to significant mortality and morbidity. While the need for efficacious novel antibiotics and antifungal is clear, the drug development pipeline has not kept pace. Most of the pharmaceutical companies have largely abandoned the antibiotic development programme because the path to market and profitability is unsure. ${ }^{20}$ The cyclic lipopeptide daptomycin, oxazolidinone linezolid and the glycylcycline tigecycline are the only three novel class of antibiotics which have been approved since the year $2000 .^{21}$ The dearth of effective treatment options ultimately leads to reintroduce old, forgotten antibiotics into their clinical practice. ${ }^{22}$ Along with CAM, polymyxins, fosfomycin, fusidic acid, cotrimoxazole, aminoglycosides are the other old antibiotics which are revamping as valuable alternatives for the treatment of difficultto-treat medical conditions. ${ }^{23}$ From the result obtained, it is evident that the CAM-LEO-PCL-P NCs delivery system of a "hydrophobic drug in essential oil" is an advantageous drug delivery system for various applications where we can exploit the therapeutic potential of both the agents i.e. drug and essential oil. Almost all of the chemical compounds identified in Cymbopogon flexuous essential oil reported having broad spectrum antimicrobial activity. The prepared NCs are having the following advantageous characteristics: small particle size (124.8 $\mathrm{nm}$ ), suitable size distribution, high drug loading, moderate preparation condition and sustained drug-release properties. The sustained release characteristics of the prepared nanocapsule system could be an advantageous delivery system as long circulating nanocarriers in drug delivery applications. Though CAM-LEO-PCL-P NCs showed zeta potential of $-22.4 \mathrm{mV}$, this nano capsule suspension maintained its stability even after six months of storage. Unknown properties like temperature dependent viscosity and refractive index (RI) of the tested solution plays crucial role in the zeta potential analysis. In multicomponent suspensions like nano capsules having various dissolved solids and residual reactants which can affect both the RI and viscosity of the dispersant and ultimately effects the absolute zeta potential. ${ }^{18}$ The bioactivity is influenced by surface properties and particle size in terms of in vitro drug release, cellular uptake, cytotoxicity, interaction with the bacterial cell of these nanocapsules. These physical 
parameters also influence in vivo pharmacokinetics and bio distribution and thereby the therapeutic index of the encapsulated drug. ${ }^{24}$

The prepared nano formulation showed significantly enhanced antimicrobial activity against nineteen bacterial strains including ten clinical isolates of MRSA, compared with the free CAM and free LEO. Before performing in vivo experiments, it is necessary to evaluate the compatibility of therapeutic agents towards mammalian cells to study the possible toxic effects. In this study, the nano encapsulation of the CAM and LEO resulted in significant decrease in the cytotoxicity of CAM against human RBC, WBC, L929 mouse fibroblast and human dermal fibroblast cells. The CAM-LEO-PCL-P NCs exhibited a significant enhancement in in vitro anti-MRSA activity compared to free CAM and free LEO. The significant enhancement in the antibacterial action is due to the combined synergistic effect of stabilized nanocapsule CAM and LEO. Being multicomponent in its chemical nature and alternative mechanisms of action, LEO can affect many microbial structures concurrently. It is established that essential oil having the unique ability to combat drug resistance by efflux pump inhibitor activity of EOs. So incorporation of this essential oil as the oily phase of nano-capsule preparation it will give an advantage of reclaiming the antimicrobials which are not active against drug resistance strains. The fabricated nano form demonstrated significant in vivo efficacy of CAM-LEO-PCL-P NCs against MRSA infected in burn wounds, as the NCs were able to penetrate into thermally injured dermal tissue and speed up wound closure by significantly reduce MRSA burden. Instinctively, CAM-LEO-PCL-P NCs provides localized coverage and facilitate minimized exposure of CAM to mammalian cells systemically resulted in the decrease in CAM's deleterious side effects and allows efficient targeting of MRSA; colonizing beneath the skin. Unlike systemic therapeutic agents, topically administered NCs avoid end-organ accumulation as it is not subjected to variable metabolism pathways for drug excretion. Furthermore, there is a possibility of systemic dispersion of topically applied CAM-LEO-PCL-P NCs when applied to a fullthickness burn wound, which required further evaluation.

\section{Conclusion}

The findings of the present study demonstrate a feasible nano capsular approach towards successful restoration of CAM. It also explains the co-encapsulation procedure of LEO and CAM in a biocompatible polymer. This nanocapsular strategy holds an immense potential to deliver hydrophobic drugs and essential oils for topical applications. The fabricated CAMLEO-PCL-P NCs exhibits broad spectrum antimicrobial activity against Candida species and bacterial strains (Gram +ve \& Gram -ve) including clinical MRSA isolates. This system was found to be biocompatible towards mammalian cells. This multicomponent drug delivery system effectively eradicates the MRSA-CA co-infection on chronic burn wounds. The graphical representation on proposed mechanism of action of the study has been given in Fig. 8. This study opens up an alternative, accelerated and cost-effective approach to develop new treatment options
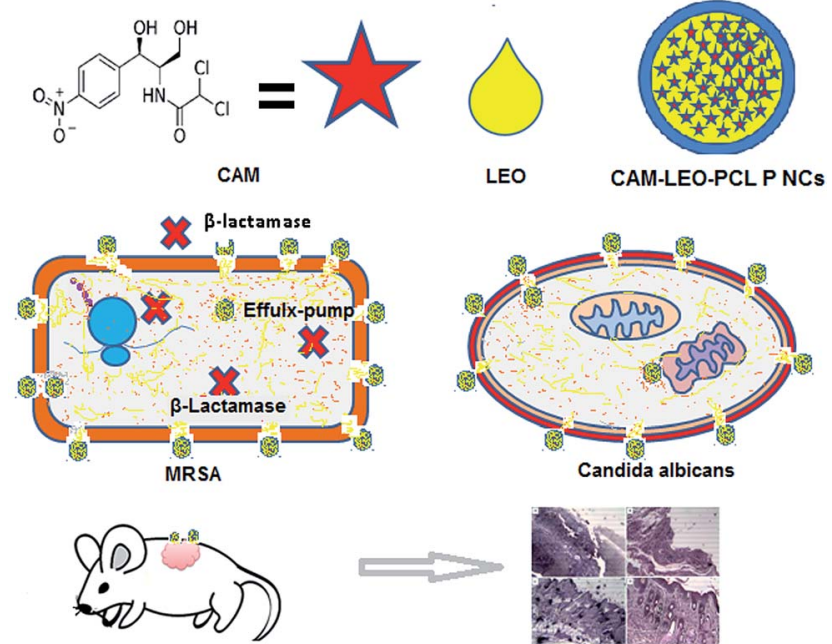

Topical delivery of CAM-LEO-PCL P NCs

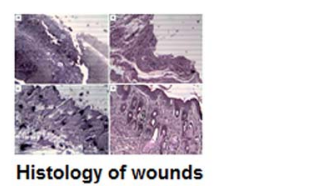

Fig. 8 Graphical representation for mechanism of action of the study.

for various infections of chronic wounds. Further in vivo investigations including pharmacokinetics, bio distribution, pharmacodynamics and toxicity studies are going on in our laboratory.

\section{Conflict of interest}

A portion of the work reported in this article has been used to file an Indian patent application (201631008544) dated $11^{\text {th }}$ March 2016.

\section{Acknowledgements}

The authors are grateful for the support from the Director of the Institute of Advanced Study in Science and Technology (IASST) and the Department of Science and Technology, Government of India for the funding of the research. Mr Kandimalla would like to thank Department of Biotechnology (DBT), Govt. of India for the support through fellowship.

\section{References}

1 D. Church, S. Elsayed, O. Reid, B. Winston and R. Lindsay, Clin. Microbiol. Rev., 2006, 19, 403-434.

2 W. M. El-Refaie, Y. S. R. Elnaggar, M. A. El-Massik and O. Y. Abdallah, Int. J. Pharm., 2015, 486(1-2), 88-98.

3 F. Jacobsen, C. Fisahn, M. Sorkin, I. Thiele, T. Hirsch, I. Stricker, T. Klaassen, A. Roemer, B. Fugmann and L. Steinstraesser, Antimicrob. Agents Chemother., 2011, 55, 2325-2334.

4 T. Katakura, M. Yoshida, D. N. Herndon and F. Suzuki, Clin. Exp. Immunol., 2005, 142, 419-425.

5 M. E. Shirtliff, B. M. Peters and M. A. Jabra-Rizk, FEMS Microbiol. Lett., 2009, 299, 1-8.

6 L. M. Schlecht, B. M. Peters, B. P. Krom, J. A. Freiberg, G. M. Hansch, S. G. Filler, M. A. Jabra-Rizk and M. E. Shirtliff, Microbiology, 2015, 161, 168-181. 
7 A. Felk, M. Kretschmar, A. Albrecht, M. Schaller, S. Beinhauer, T. Nichterlein, D. Sanglard, H. C. Korting, W. Schäfer and B. Hube, Infect. Immun., 2002, 70, 3689-3700. 8 M. Fayyaz, I. A. Mirza, Z. Ahmed, S. A. Abbasi, A. Hussain and S. Ali, J. Coll. Phys. Surg. Pakistan, 2013, 23, 637-640.

9 M. A. Szabó, G. Z. Varga, J. Hohmann, Z. Schelz, E. Szegedi, L. Amaral and J. Molnár, Phytother. Res., 2010, 24, 782-786.

10 Y. W. Wang, W. C. Zeng, P. Y. Xu, Y. J. Lan, R. X. Zhu, K. Zhong, Y. N. Huang and H. Gao, Int. J. Mol. Sci., 2012, 13, 3382-3393.

11 E. Eroglu, P. M. Tiwari, A. B. Waffo, M. E. Miller, K. Vig, V. A. Dennis and S. R. Singh, Int. J. Nanomed., 2013, 8, 1403-1415.

12 E. Larrañeta, M. T. C. McCrudden, A. J. Courtenay and R. F. Donnelly, Pharm. Res., 2016, 33, 1055-1073.

13 I. L. Liakos, F. A. Garzoni, C. Bonferoni, A. Scarpellini, V. Brunetti, R. Carzino, P. Bianchini, P. P. Pompa and A. Athanassiou, Int. J. Pharm., 2016, 510(2), 508-515.

14 A. Baranowska-Korczyc, A. Warowicka, M. JasiurkowskaDelaporte, B. Grześkowiak, M. Jarek, B. M. Maciejewska, J. Jurga-Stopa and S. Jurga, $R S C A d v .$, 2016, 6, 19647-19656.
15 A. Herman and A. P. Herman, Journal of Pharmacy and Pharmacology, 2014, 67, 473-485.

16 M. N. Boukhatem, M. A. Ferhat, A. Kameli, F. Saidi and H. T. Kebir, Libyan Journal of Medicine, 2014, 9, 1-10.

17 M. G. Miguel, Molecules, 2010, 15, 9252-9287.

18 G. V. Lowry, R. J. Hill, S. Harper, A. F. Rawle, C. O. Hendren, F. Klaessig, U. Nobbmann, P. Sayre and J. Rumble, Environ. Sci.: Nano, 2016, 3, 953-965.

19 T. Braine, Bull. W. H. O., 2011, 89, 88-89.

20 Z. Xing, J. Gauldie, G. Cox, H. Baumann, M. Jordana, X. F. Lei and M. K. Achong, J. Clin. Invest., 1998, 101, 311-320.

21 P. Chithra, G. B. Sajithlal and G. Chandrakasan, J. Ethnopharmacol., 1998, 59, 179-186.

22 F. B. Sime, M. S. Roberts and J. A. Roberts, Clin. Microbiol. Infect., 2015, 21, 886-893.

23 F. Jacobsen, C. Fisahn, M. Sorkin, I. Thiele, T. Hirsch, I. Stricker, T. Klaassen, A. Roemer, B. Fugmann and L. Steinstraesser, Antimicrob. Agents Chemother., 2011, 55, 2325-2334.

24 A. Bhaskar and V. Nithya, Indian J. Pharmacol., 2012, 44, 694698. 